\title{
Diagnostic accuracy of Xpert MTB/RIF assay for musculoskeletal tuberculosis: a meta-analysis
}

\section{Hai Wen \\ Pengzhi Li \\ Hong $\mathrm{Ma}$ \\ Guohua Lv}

Department of Spine Surgery, The Second Xiangya Hospital of Central South University, Changsha, People's Republic of China
Correspondence: Guohua Lv Department of Spine Surgery, The Second Xiangya Hospital of Central South University, 139 Renmin Middle Road, Changsha 4I00II, People's Republic of China

$\mathrm{Tel} / \mathrm{Fax}+8673184327198$

Email spinelgh@।63.com
This article was published in the following Dove Press journal:

Infection and Drug Resistance

28 September 2017

Number of times this article has been viewed

Objectives: Xpert MTB/RIF assay, a rapid and automated real-time nucleic acid amplification test, has been reported for the diagnosis of musculoskeletal tuberculosis (TB) in current years. This meta-analysis aims to determine the diagnostic accuracy of Xpert for the detection of musculoskeletal TB and rifampicin (RIF) resistance.

Methods: We searched PubMed, Embase, China National Knowledge Infrastructure, and Wanfang for original articles published up to 1st June 2017 to identify studies in which the Xpert assay was applied to diagnose musculoskeletal TB. Pooled estimates were calculated using a random-effects model or a fixed-effects model according to heterogeneity. Summary receiver operating characteristic curves and the area under the curve (AUC) were used to summarize overall diagnostic performance. Deeks' test was performed to evaluate potential publication bias. Results: Twelve studies were identified with a pooled sensitivity and specificity of respectively 0.81 (95\% confidence interval [CI] 0.78-0.83) and 0.83 (95\% CI 0.80-0.86) of Xpert for the diagnosis of musculoskeletal TB. Xpert was highly sensitive $(0.89,95 \%$ CI $0.79-0.95)$ and highly specific $(0.96,95 \%$ CI $0.92-0.98)$ in detecting RIF resistance. AUC (over 0.9) suggested a relatively high level of overall diagnostic accuracy of Xpert for detecting musculoskeletal TB and RIF resistance. Prevalence and reference standard were indicated to be sources of heterogeneity between studies. No publication bias was found.

Conclusion: This study provides available evidence of the rapid and effective role of Xpert in diagnosing musculoskeletal TB and detecting RIF resistance.

Keywords: Xpert, diagnostic accuracy, musculoskeletal tuberculosis, meta-analysis, rifampicin resistance

\section{Introduction}

Tuberculosis (TB) remains to be one of the predominant infectious diseases and ranks as a leading cause of infection-related mortality worldwide. ${ }^{1,2}$ Besides the major pulmonary TB (PTB), extrapulmonary sites (EPTB) are often involved during the initial latent phase of the infection. ${ }^{3}$ EPTB accounts for $10 \%-42 \%$ of TB cases, $10 \%-25 \%$ of which are musculoskeletal TB. ${ }^{4,5}$ Almost half of all cases of musculoskeletal TB have vertebral involvement. ${ }^{6}$ Joint destruction, growth arrest, and contractures in large joints, as well as deformity with neurologic compromise in spinal disease, may result in long-term morbidity and disability, ${ }^{7,8}$ particularly in multidrug-resistant (MDR) cases of TB, which are increasing especially in the developing countries. Therefore, more accurate and timely tests compared with conventional diagnostic approaches are critical for musculoskeletal TB to enable early effective treatment that is associated with reduced deformity and enhanced outcome. 
Molecular tests have been investigated previously to identify Mycobacterium tuberculosis and mutations conferring resistance to rifampicin (RIF) and/or isoniazid. ${ }^{910}$ Xpert MTB/RIF (Xpert/GeneXpert) assay, a rapid and automated real-time nucleic acid amplification test that can detect RIF susceptibility within $2 \mathrm{~h}$, has been endorsed by the World Health Organization (WHO) for the diagnosis of PTB. ${ }^{11}$ According to a recent meta-analysis, the pooled sensitivity and specificity of Xpert in diagnosing children's PTB were $68 \%$ and $100 \%$, respectively. ${ }^{12}$ The WHO also recommended Xpert for the diagnosis of EPTB in 2013, ${ }^{13}$ and this technology was indicated by another meta-analysis to have high specificity but relatively low sensitivity in the detection of EPTB.${ }^{14}$ Applications of Xpert for musculoskeletal TB cases have been reported in current years, but the data are very limited. Held et a ${ }^{15}$ reported that Xpert had a sensitivity of $95.6 \%$ and a specificity of $96.2 \%$ in 71 adult spinal samples compared with culture or histology as a reference standard. Gu et a ${ }^{16}$ performed Xpert in 60 cases of bone and joint TB and detected a sensitivity of $82 \%$ and a specificity of $100 \%$ using composite reference standard (CRS). In consideration of the variable results, we performed this updated metaanalysis to assess the overall diagnostic accuracy of the Xpert assay in the detection of musculoskeletal TB.

\section{Methods}

\section{Search strategy and study selection}

This meta-analysis was conducted according to the criteria of the Preferred Reporting Items for Systematic Reviews and Meta-Analyses group. ${ }^{17}$ A systematic search was performed using PubMed, Embase, China National Knowledge Infrastructure, and Wanfang for original articles published up to 1 st June 2017 to identify studies in which the Xpert assay was applied to diagnose musculoskeletal TB. The following search terms were used: "Xpert" OR "GeneXpert" AND “tuberculosis" OR “TB” AND "bone” OR “joint” OR "spine". There were no language restrictions. References within selected articles were also reviewed to identify additional relevant studies.

Titles and abstracts of all studies were independently screened, and afterwards, full-text publications of relevant articles were read by two investigators (H Wen and P Li). Disagreements were resolved by consensus. A study was included when it met the following inclusion criteria: an original research article written in English or Chinese; the Xpert assay was used for the diagnosis of musculoskeletal TB, and the reference standard was described; and sufficient data including true-positive, false-positive, false-negative, and true-negative results of the Xpert assay were provided.

\section{Data extraction and quality assessment}

The same two reviewers independently extracted data from each included study, and discrepancies were resolved by consensus. The retrieved data included author, publication year, country, sample size, specimen types, reference standard, and numbers of true-positive, false-positive, false-negative, and true-negative results. The methodological quality of each study was assessed using the validated Quality Assessment of Diagnostic Accuracy Studies (QUADAS) tool. ${ }^{18}$

\section{Statistical analysis}

We used Meta-DiSc 1.4 (XI Cochrane Colloquium, Barcelona, Spain) to estimate the following data of individual study: sensitivity, specificity, positive likelihood ratio (PLR), negative likelihood ratio (NLR), and diagnostic odds ratio (DOR) together with $95 \%$ confidence intervals (95\% CIs). A summary receiver operating characteristic (SROC) curve was plotted on the basis of pooled sensitivity and specificity, and the overall diagnostic performance of Xpert was displayed by the area under the curve (AUC). Heterogeneity between studies was evaluated using the $Q$ test and $I^{2}$ statistics, according to which pooled indices across studies were calculated using a random-effects model or a fixed-effects model. Deeks' funnel plot was generated using STATA 12.0 (Stata Corp., College Station, TX, USA) to detect the potential publication bias. ${ }^{19}$ All statistical tests were two-sided, and $p$ values less than 0.05 were considered statistically significant.

\section{Results}

\section{Search result and study characteristics}

Our search yielded a total of 466 citations, 12 of which were finally included in the current meta-analysis based on the inclusion criteria (Figure 1 and Table 1). ${ }^{15,16,20-29}$ The included studies were published between 2014 and 2017. Data came from the People's Republic of China, South Africa, India, and Indonesia, all of which are countries with high TB burden and low/middle income. The studies totally enrolled 1690 specimens with an average sample size of 141 (range, 49-338). All the studies described the performance of the Xpert assay in suspected musculoskeletal TB samples which were tissue or pus taken from bones, joints, or spine, but the individual reference standard to assess the diagnostic accuracy of Xpert was not consistent, including mycobacterial culture only (141 specimens), culture or histology (586 specimens), and CRS (963 specimens). The CRS included all patients who have 


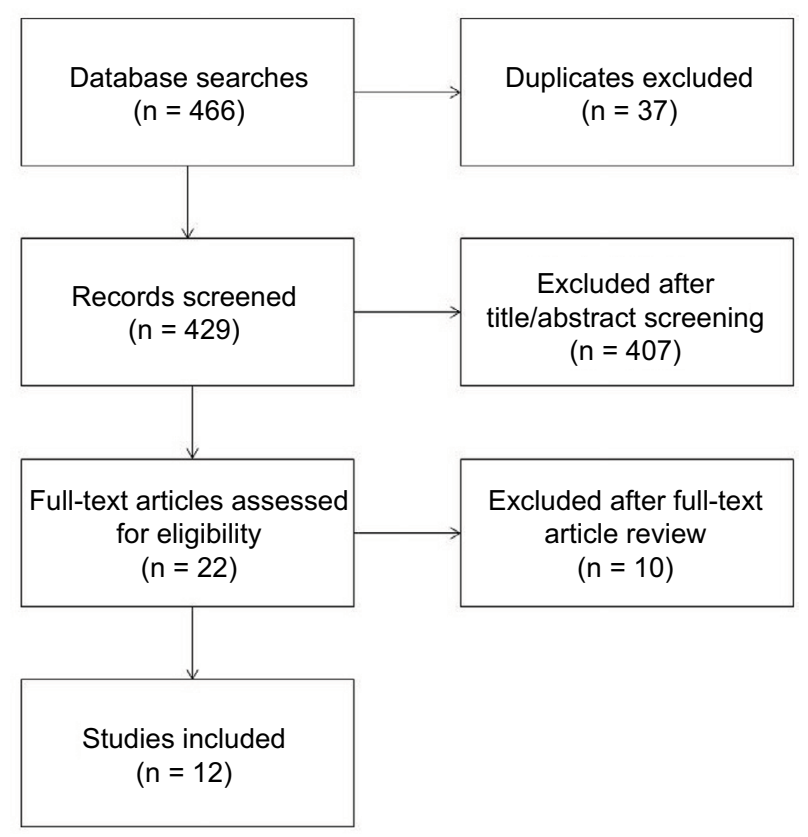

Figure I Flow chart of study selection in this meta-analysis.

improved both clinically and radiologically after empirical antituberculous therapy where the results of culture, smear, and histology might be positive or negative for TB. ${ }^{20}$ Moreover, the mean score of the 12 studies was 9.42 (range, 9-11) according to the criteria of QUADAS (Table 1).

\section{Diagnostic accuracy of Xpert for musculoskeletal TB detection}

Pooled sensitivity and specificity of Xpert in musculoskeletal TB detection are shown in Figure 2A. Among the 12 included studies, sensitivity ranged from 0.69 to 1.00 (pooled 0.81 , 95\% CI $0.78-0.83$ ), and specificity ranged from 0.17 to 1.00 (pooled $0.83,95 \%$ CI $0.80-0.86$ ). Additionally, pooled parameters including PLR (10.90, 95\% CI 3.11-38.20), NLR $(0.22,95 \%$ CI $0.19-0.25)$, and DOR (50.86, 95\% CI $22.00-117.56)$ were calculated. Reflected by $I^{2}$ statistics of the indices $(56.5 \%$ for sensitivity and $94.2 \%$ for specificity), there existed heterogeneity in diagnostic performance between studies. An SROC curve displaying sensitivity against 1 - specificity from individual study was plotted for Xpert in musculoskeletal TB, with an AUC of 0.9182 (Figure 3A), suggesting high overall diagnostic accuracy of the Xpert assay in detection of musculoskeletal TB.

\section{Diagnostic accuracy of Xpert in detection of RIF-resistant musculoskeletal TB}

There were five studies which reported the results of RIF resistance in culture-positive musculoskeletal TB clearly

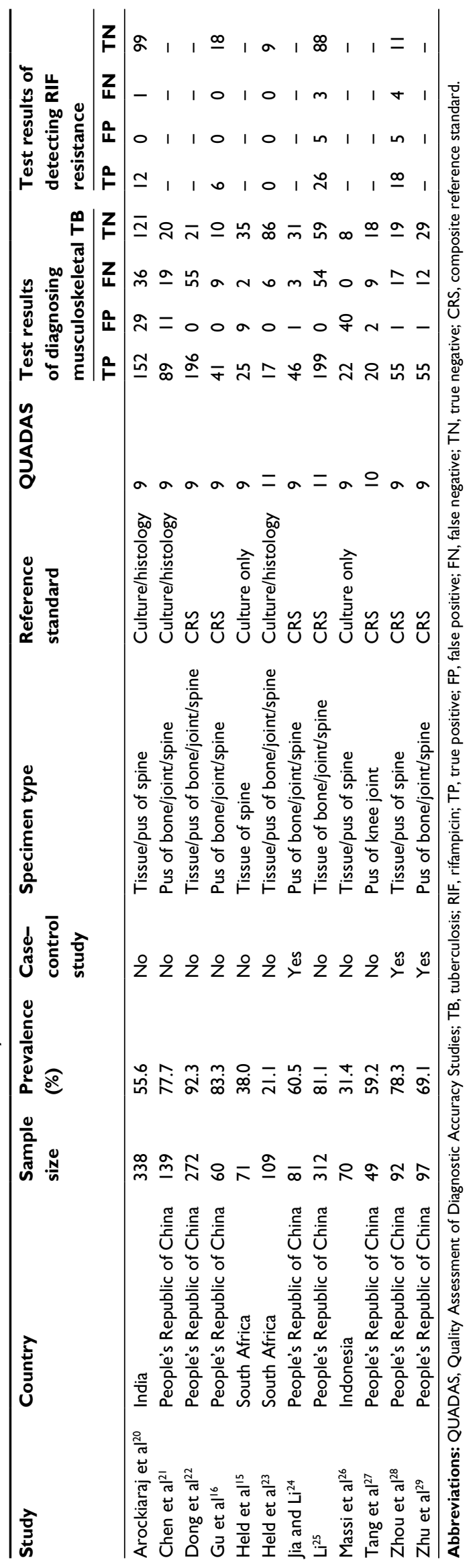




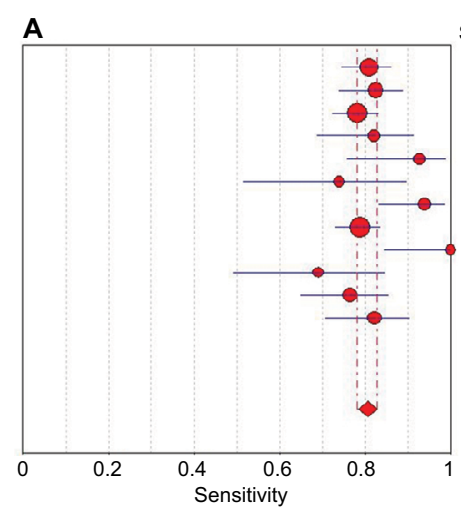

8

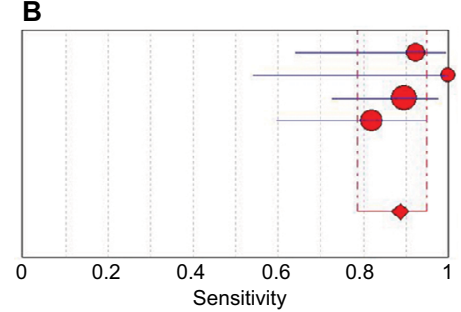

Sensitivity $(95 \% \mathrm{Cl})$

$0.81 \quad(0.74-0.86)$

$0.82 \quad(0.74-0.89)$

$0.78 \quad(0.72-0.83)$

$0.82(0.69-0.91)$

$0.93(0.76-0.99)$

$0.74 \quad(0.52-0.90)$

$0.94 \quad(0.83-0.99)$

$0.79 \quad(0.73-0.84)$

$1.00 \quad(0.85-1.00)$

$0.69 \quad(0.49-0.85)$

$0.82(0.71-0.90)$

Pooled sensitivity $=0.81(0.78-0.83)$

$\mathrm{Chi}^{2}=25.26 ; d f=11(p=0.0083)$

Inconsistency $\left(I^{2}\right)=56.5 \%$

Sensitivity $(95 \% \mathrm{Cl})$

$0.92(0.64-1.00)$

$1.00 \quad(0.54-1.00)$

$0.90 \quad(0.73-0.98)$

$0.82(0.60-0.95)$

Arockiaraj et $\mathrm{al}^{20}$

$\mathrm{Li}^{25}$ et $\mathrm{al}^{16}$

Zhou et $\mathrm{al}^{28}$

Pooled sensitivity $=0.89(0.79-0.95)$

$\mathrm{Chi}^{2}=2.55 ; d f=3(p=0.4663)$

Chi ${ }^{2}=2.55 ; d f=3(p=0.463$
Inconsistency $\left(I^{2}\right)=0.0 \%$
Arockiaraj et al ${ }^{20}$

Chen et $\mathrm{al}^{21}$

Dong et $\mathrm{al}^{22}$

Held et al ${ }^{15}$

Held et al ${ }^{23}$

$\mathrm{Jia}$ and $\mathrm{Li}^{24}$

Massi et al26

Tang et $\mathrm{al}^{27}$

Tang et al 27

Chou et al28

Pooled specificity $=0.83(0.80-0.86)$ $\mathrm{Chi}^{2}=189.35 ; d f=11(p=0.0000)$ Inconsistency $\left(I^{2}\right)=94.2 \%$

Specificity $(95 \% \mathrm{Cl})$

$0.81 \quad(0.73-0.87)$

$0.65 \quad(0.45-0.81)$

$1.00(0.84-1.00)$

$1.00(0.69-1.00)$

$0.80 \quad(0.65-0.90)$

$1.00 \quad(0.96-1.00)$

$0.97 \quad(0.84-1.00)$

$1.00(0.94-1.00)$

$0.90 \quad(0.68-0.99)$

$0.95(0.75-1.00)$

$0.97 \quad(0.83-1.00)$



Specificity $(95 \% \mathrm{Cl})$

$1.00 \quad(0.96-1.00)$

$\begin{array}{ll}1.00 & (0.81-1.00) \\ 0.95 & (0.88-0.98)\end{array}$

$0.95 \quad(0.88-0.98)$

0.69 (0.41-0.89)

Pooled specificity $=0.96(0.92-0.98)$ $\mathrm{Chi}^{2}=23.08 ; d f=3(p=0.0000)$ Inconsistency $\left(I^{2}\right)=87.0 \%$

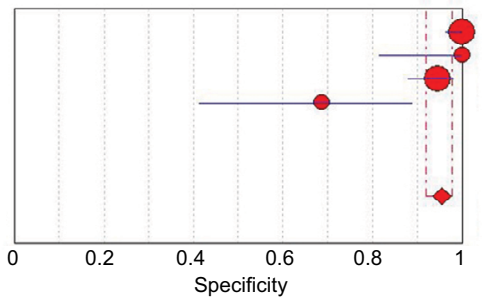

Figure 2 Analysis of sensitivity and specificity of Xpert MTB/RIF assay. Forest plots of sensitivity and specificity of the assay in (A) diagnosing musculoskeletal TB and (B) detecting RIF-resistance. Point estimates of sensitivity and specificity from each study are shown as solid circles. Error bars indicate $95 \%$ confidence interval.

Abbreviation: $\mathrm{Cl}$, confidence interval.

A



B

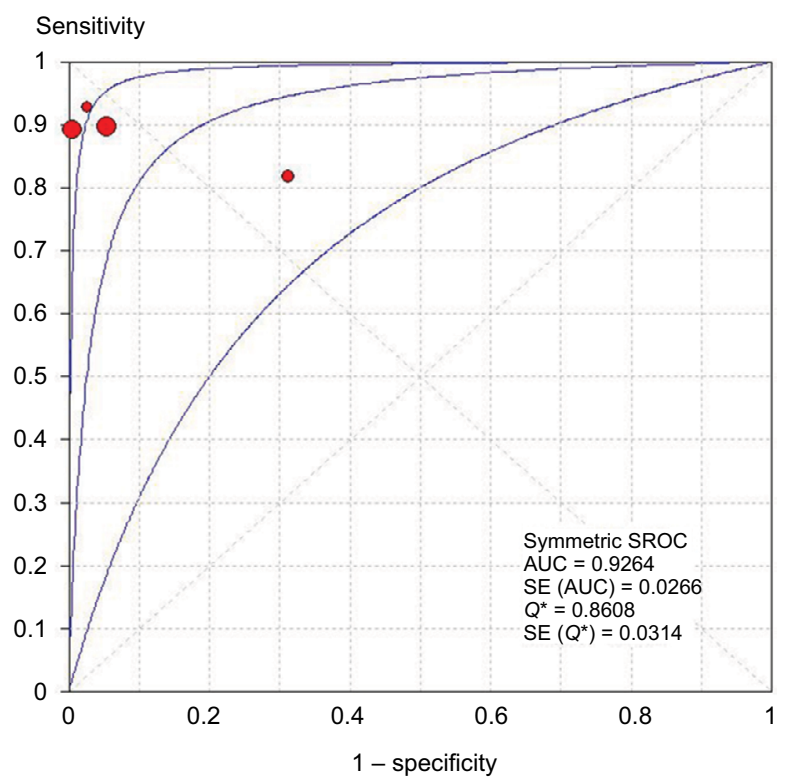

Figure 3 SROC curves of Xpert for the (A) diagnosis of musculoskeletal TB and (B) detection of RIF resistance. The size of each solid circle represents the sample size of individual study. The regression SROC curves summarize the overall diagnostic accuracy.

Abbreviations: SROC, summary receiver operating characteristic; TB, tuberculosis; RIF, rifampicin; AUC, area under the curve; SE, standard error.

(Table 1). ${ }^{16,20,23,25,28}$ Among them, one study ${ }^{23}$ was not included in the analysis due to no positive results of Xpert for detection of RIF-resistant musculoskeletal TB. The pooled sensitivity $(0.89,95 \%$ CI $0.79-0.95)$ and specificity $(0.96,95 \%$ CI 0.92-0.98) in diagnosing RIF-resistant musculoskeletal TB for four studies are displayed in Figure 2B. The pooled PLR, NLR, and DOR were 16.00 (95\% CI 2.98-86.04), 0.13
(95\% CI 0.06-0.25), and 128.60 (95\% CI 13.69-1208.09), respectively. The SROC curve is illustrated in Figure 3B, and the AUC was 0.9264.

\section{Heterogeneity and subgroup analysis}

Indicated by the Spearman correlation coefficient $(0.483$, $p=0.112$ ), heterogeneity between studies was not correlated 
with threshold effect. We hypothesized that the most likely factors causing heterogeneity were as follows: sample size, prevalence, study design, specimen type, reference standard, and QUADAS scores. Heterogeneity analysis showed that prevalence and reference standard were the probable sources of heterogeneity (both $p<0.05$ ). Therefore, we stratified the 12 studies into subgroups by prevalence and reference standard. As shown in Table 2, the sensitivity and specificity of Xpert in studies with lower prevalence $(<50 \%)$ respectively elevated and declined compared with those with higher prevalence ( $\geq 50 \%$ ). Besides, Xpert was most sensitive $(0.96,95 \%$ CI $0.86-1.00)$ and least specific $(0.47,95 \%$ CI $0.36-0.57)$ in studies using culture as the reference standard. In contrast, Xpert was most specific $(0.97,95 \%$ CI $0.94-0.99)$ and least sensitive $(0.79,95 \% \mathrm{CI} 0.76-0.82)$ in studies using CRS as the reference standard. Reflected by $I^{2}(46.0 \%$ for sensitivity and $22.4 \%$ for specificity), additionally, there was no significant heterogeneity of the diagnostic accuracy of Xpert in studies using CRS as the reference standard.

\section{Publication bias}

Deeks' funnel plot asymmetry test was performed to evaluate potential publication bias of included studies. In spite of some asymmetry shown in the funnel plots due to the limited quantity of studies (Figure 4), the $p$ value (0.621) of Deeks' test was not statistically significant, indicating low likelihood of publication bias.

\section{Discussion}

Musculoskeletal TB is the third most common type of EPTB after pleural and lymphatic disease, and the spine and weight-bearing joints are the most vulnerable sites of infection. ${ }^{6}$ Although identification of $M$. tuberculosis and drug susceptibility tests remain the gold standard for TB diagnosis, pathogen culturing is time-consuming and owns a relatively high false-negative rate, ${ }^{30}$ together with the increasing incidence of MDR-TB, leading to a pressing need for more timely and effective diagnostic methods. A rising and promising rapid test, Xpert MTB/RIF assay, has been

Table 2 Subgroup analysis of the results of Xpert for detecting musculoskeletal TB

\begin{tabular}{|c|c|c|c|c|c|c|}
\hline Variables & Number of studies & Sensitivity $(95 \% \mathrm{Cl})$ & $I^{2}$ & Specificity $(95 \% \mathrm{Cl})$ & $P^{2}$ & AUC \\
\hline \multicolumn{7}{|l|}{ Prevalence (\%) } \\
\hline$\geq 50$ & 9 & $0.80(0.77-0.82)$ & $32.2 \%$ & $0.88(0.84-0.91)$ & $83.5 \%$ & 0.8820 \\
\hline$<50$ & 3 & $0.89(0.79-0.95)$ & $79.1 \%$ & $0.72(0.65-0.79)$ & $98.4 \%$ & 0.9568 \\
\hline \multicolumn{7}{|l|}{ Reference standard } \\
\hline Culture only & 2 & $0.96(0.86-1.00)$ & $59.2 \%$ & $0.47(0.36-0.57)$ & $97.5 \%$ & NE \\
\hline Culture/histology & 3 & $0.81(0.76-0.85)$ & $0.0 \%$ & $0.85(0.80-0.89)$ & $94.7 \%$ & 0.8780 \\
\hline CRS & 7 & $0.79(0.76-0.82)$ & $46.0 \%$ & $0.97(0.94-0.99)$ & $22.4 \%$ & 0.9429 \\
\hline
\end{tabular}

Abbreviations: TB, tuberculosis; $\mathrm{Cl}$, confidence interval; $\mathrm{AUC}$, the area under the curve; NE, not estimable; CRS, composite reference standard.



Figure 4 Deeks' funnel plot indicating no significant risk of publication bias $(p=0.621)$. Solid circles represent each study included in the meta-analysis. Abbreviation: ESS, effective sample size. 
identified to diagnose TB and detect RIF resistance rapidly and effectively. ${ }^{910}$ This assay was reported to detect as low as 131 colony forming units per milliliter of M. tuberculosis culture from a specimen. ${ }^{31}$ In the current study, we evaluated the diagnostic accuracy of Xpert for the detection of musculoskeletal TB and RIF resistance.

Our data showed that the pooled sensitivity and specificity of Xpert in detecting musculoskeletal TB were $81 \%$ and $83 \%$, respectively. The efficacy of Xpert is lower (sensitivity of $89 \%$ and specificity of 99\%) in the diagnosis of PTB according to a previous meta-analysis, ${ }^{32}$ which may be owing to less content of M. tuberculosis in musculoskeletal specimens compared with sputum from patients with PTB. ${ }^{20}$ Penz et a ${ }^{14}$ described a pooled sensitivity and specificity of respectively $77 \%$ and $97 \%$ of Xpert in the detection of EPTB in a meta-analysis including 36 studies. Relatively homogeneous tested samples and less included studies, especially some describing comparatively low specificity, ${ }^{25,26}$ may account for the reduced pooled specificity in our study. In addition, a pooled PLR of 10.90 and a pooled NLR of 0.22 indicate that patients with positive or negative Xpert results have a 10.90-fold higher or a 0.22 -fold lower chance of suffering from musculoskeletal TB than not. These data may not be convincing enough for clinical use. Nevertheless, a pooled DOR of 50.86 indicates significant discriminant effect, and an AUC of 0.9182 suggests a relatively high level of overall diagnostic accuracy of Xpert for detecting musculoskeletal TB. It is generally known that simultaneous assessment of RIF resistance is a crucial advantage of Xpert. This test was revealed by our analysis to have high pooled sensitivity $(89 \%)$ and specificity (96\%) in the diagnosis of RIF-resistant musculoskeletal TB, which is consistent with the high efficacy of Xpert in detecting RIF-resistant PTB reported by Chang et al. ${ }^{33}$ Moreover, other parameters including pooled PLR (16.00), pooled NLR (0.13), pooled DOR (128.60), and AUC (0.9264) support that Xpert meets the requirements of effective detection of RIF resistance.

Expectedly, significant heterogeneity of results between studies was observed, whereas it was not correlated with threshold effect. Due to the limited data of included studies in this meta-analysis, we restricted meta-regression analysis to six factors mentioned above, and attributed the heterogeneity to prevalence and reference standard. It was indicated by subgroup analysis that in studies with lower prevalence of musculoskeletal TB, Xpert showed increased sensitivity and reduced specificity, which could be explained by relatively less positive and more negative results detected by the reference standard used in these studies. Also, diagnostic accuracy varied significantly using different reference standards. Although mycobacterial culture has been widely accepted as a gold standard for diagnosing TB, its relatively high false-negative rate may raise negative results and decrease positive results. Hence, high sensitivity (96\%) and low specificity (47\%) of Xpert were pooled when using this gold standard only. We meanwhile observed a much higher pooled specificity (97\%) of Xpert when CRS was used as the reference standard, which was probably because of reduced false-negative results according to CRS.

Several limitations of the current meta-analysis should be considered. Lack of included studies, particularly those recording the diagnostic performance of Xpert for RIF resistance, may limit the statistical power for drawing definite conclusions about the efficacy of Xpert in detecting musculoskeletal TB and RIF-resistant cases. Also, three included studies ${ }^{24,28,29}$ designed as case-control probably overvalued the diagnostic accuracy of Xpert as a result of diagnostic suspicion bias. In addition, most studies did not mention the information on blinding, and sample collection and processing were variable across the studies. The pooled estimates should be interpreted with caution in consideration of existing heterogeneity among the studies.

In conclusion, our study demonstrates that Xpert MTB/ RIF assay has high sensitivity and specificity in the diagnosis of musculoskeletal TB and detection of RIF resistance, providing useful information for clinical decision-making. More efforts should be made to define the accuracy of this promising test for diagnosing musculoskeletal TB in the future.

\section{Acknowledgments}

The authors acknowledge the grants from the Fundamental Research Funds for the Central Universities of Central South University (No. 2016zzts149).

\section{Disclosure}

The authors report no conflicts of interest in this work.

\section{References}

1. Lienhardt C, Glaziou P, Uplekar M, Lönnroth K, Getahun H, Raviglione M. Global tuberculosis control: lessons learnt and future prospects. Nat Rev Microbiol. 2012;10(6):407-416.

2. Giacomini G, Miranda JR, Pavan AL, et al. Quantification of pulmonary inflammatory processes using chest radiography: tuberculosis as the motivating application. Medicine (Baltimore). 2015;94(26):e1044.

3. Reuter H, Wood R, Schaaf H, et al. Overview of extrapulmonary tuberculosis in adults and children. 2009;377-390. Available from: http:// www.sciencedirect.com/science/article/pii/B9781416039884000342. Accessed September 18, 2017.

4. WorldHealth Organization. Globaltuberculosis report-2014.Available from: http://apps.who.int/iris/bitstream/10665/137094/1/9789241564809_eng. pdf. Accessed September 18, 2017. 
5. Trecarichi EM, Di Meco E, Mazzotta V, Fantoni M. Tuberculous spondylodiscitis: epidemiology, clinical features, treatment, and outcome. Eur Rev Med Pharmacol Sci. 2012;16 Suppl 2:58-72.

6. Leonard MK, Blumberg HM. Musculoskeletal tuberculosis. Microbiol Spectr. 2017;5(2):371-392.

7. Rajasekaran S, Shanmugasundaram TK. Prediction of the angle of gibbus deformity in tuberculosis of the spine. J Bone Joint Surg Am. 1987;69(4):503-509.

8. Watts HG, Lifeso RM. Tuberculosis of bones and joints. J Bone Joint Surg Am. 1996;78(2):288-298.

9. Barnard M, Albert H, Coetzee G, O’Brien R, Bosman ME. Rapid molecular screening for multidrug-resistant tuberculosis in a highvolume public health laboratory in South Africa. Am J Respir Crit Care Med. 2008;177(7):787-792.

10. Bwanga F, Hoffner S, Haile M, Joloba ML. Direct susceptibility testing for multi drug resistant tuberculosis: a meta-analysis. BMC Infect Dis. 2009;9:67.

11. World Health Organization. WHO endorses new rapid tuberculosis test. 2010. Available from: http://www.who.int/tb/features_archive/ new_rapid_test/en. Accessed September 18, 2017.

12. Reither K, Manyama C, Clowes P, et al. Xpert MTB/RIF assay for diagnosis of pulmonary tuberculosis in children: a prospective, multicentre evaluation. J Infect. 2015;70(4):392-399.

13. World Health Organization. Automated Real-Time Nucleic Acid Amplification Technology for Rapid and Simultaneous Detection of Tuberculosis and Rifampicin Resistance: Xpert MTB/RIF Assay for the Diagnosis of Pulmonary and Extrapulmonary TB in Adults and Children: Policy Update. Geneva: World Health Organization; 2013.

14. Penz E, Boffa J, Roberts DJ, et al. Diagnostic accuracy of the Xpert ${ }^{\mathbb{R}}$ MTB/RIF assay for extra-pulmonary tuberculosis: a meta-analysis. Int J Tuberc Lung Dis. 2015;19(3):278-284.

15. Held M, Laubscher M, Zar HJ, Dunn RN. GeneXpert polymerase chain reaction for spinal tuberculosis: an accurate and rapid diagnostic test. Bone Joint J. 2014;96-B(10):1366-1369.

16. Gu Y, Wang G, Dong W, et al. Xpert MTB/RIF and GenoType MTBDRplus assays for the rapid diagnosis of bone and joint tuberculosis. Int J Infect Dis. 2015;36:27-30.

17. Moher D, Liberati A, Tetzlaff J, Altman DG; PRISMA Group. Preferred reporting items for systematic reviews and meta-analyses: the PRISMA statement. J Clin Epidemiol. 2009;62(10):1006-1012.

18. Whiting P, Rutjes AW, Reitsma JB, Bossuyt PM, Kleijnen J. The development of QUADAS: a tool for the quality assessment of studies of diagnostic accuracy included in systematic reviews. BMC Med Res Methodol. 2003;3:25.

19. Deeks JJ, Macaskill P, Irwig L. The performance of tests of publication bias and other sample size effects in systematic reviews of diagnostic test accuracy was assessed. J Clin Epidemiol. 2005;58(9):882-893.

20. Arockiaraj J, Michael JS, Amritanand R, David KS, Krishnan V. The role of Xpert MTB/RIF assay in the diagnosis of tubercular spondylodiscitis. Eur Spine J. Epub 2017 Apr 8.
21. Chen H, Li X, Gao M, et al. [Assessment of the Xpert MTB/RIF assay for the detection of bone and joint tuberculosis]. Chin J Clinician. 2015;43(10):24-26. Available from: http://d.wanfangdata.com.cn/ Periodical/zglcys201510008. Accessed September 18, 2017. Chinese.

22. Dong W, Qin S, Lan T, et al. [Study on Xpert MTB/RIF for rapid diagnosis of bone and joint tuberculosis and detection of rifampin resistance]. Chin J Antituberc. 2017;39(4):337-341. Available from http://d.wanfangdata.com.cn/Periodical/zgflzz201704005. Accessed September 18, 2017. Chinese.

23. Held M, Laubscher M, Mears S, et al. Diagnostic accuracy of the Xpert MTB/RIF assay for extrapulmonary tuberculosis in children with musculoskeletal infections. Pediatr Infect Dis J. 2016;35(11): 1165-1168.

24. Jia W, Li Y. [The application of Xpert MTB/RIF assay for rapid diagnosis of osteoarticular tuberculosis]. Chin J Spine Spinal Cord. 2015;25(3):208-212. Available from: http://d.wanfangdata.com.cn/ Periodical/zgjzjszz201503005. Accessed September 18, 2017. Chinese.

25. Li Z. [Evaluation of Xpert MTB/RIF on osteoarticular tissue for the diagnosis of tuberculosis and rifampin resistance]. Shanxi Medical University. 2016. Available from: http://d.wanfangdata.com.cn/Thesis/ D01089297. Accessed September 18, 2017. Chinese.

26. Massi MN, Biatko KT, Handayani I, et al. Evaluation of rapid GeneXpert MTB/RIF method using DNA tissue specimens of vertebral bones in patients with suspected spondylitis TB. J Orthop. 2017;14(1) 189-191.

27. Tang K, Dong W, Lan T, et al. [Study on rapid diagnosis of knee joint tuberculosis by Xpert MTB/RIF]. Chin JAntituberc. 2016;38(4):300-304. Available from: http://d.wanfangdata.com.cn/Periodical/zgflzz201604014. Accessed September 18, 2017. Chinese.

28. Zhou Z, Wang X, Chen H, et al. [The application of GeneXpert MTB/ RIF for diagnosis of spinal tuberculosis]. Shandong Med J. 2017;57(17): 90-92. Available from: http://d.wanfangdata.com.cn/Periodical/ shandyy201717032. Accessed September 18, 2017. Chinese.

29. Zhu Z, Xia Q, Yue Y, et al. [The value of Xpert MTB/RIF in the diagnosis of bone and joint tuberculosis]. Prev Med. 2017;29(3):322-324. Available from: http://d.wanfangdata.com.cn/Periodical/zjyfyx201703033. Accessed September 18, 2017. Chinese

30. Strassburg A, Jafari C, Ernst M, Lotz W, Lange C. Rapid diagnosis of pulmonary TB by BAL enzyme-linked immunospot assay in an immunocompromised host. Eur Respir J. 2008;31(5):1132-1135.

31. Blakemore R, Story E, Helb D, et al. Evaluation of the analytical performance of the Xpert MTB/RIF assay. J Clin Microbiol. 2010;48(7): 2495-2501.

32. Steingart KR, Schiller I, Horne DJ, et al. Xpert ${ }^{\mathbb{R}}$ MTB/RIF assay for pulmonary tuberculosis and rifampicin resistance in adults. Cochrane Database Syst Rev. 2013;(1):CD009593.

33. Chang K, Lu W, Wang J, et al. Rapid and effective diagnosis of tuberculosis and rifampicin resistance with Xpert MTB/RIF assay: a metaanalysis. J Infect. 2012;64(6):580-588.
Infection and Drug Resistance

\section{Publish your work in this journal}

Infection and Drug Resistance is an international, peer-reviewed openaccess journal that focuses on the optimal treatment of infection (bacterial, fungal and viral) and the development and institution of preventive strategies to minimize the development and spread of resistance. The journal is specifically concerned with the epidemiology of antibiotic
Dovepress

resistance and the mechanisms of resistance development and diffusion in both hospitals and the community. The manuscript management system is completely online and includes a very quick and fair peerreview system, which is all easy to use. Visit http://www.dovepress.com/ testimonials.php to read real quotes from published authors. 